\title{
Examination of injection moulded thermoplastic maize starch
}

\author{
T. Tábi, J. G. Kovács* \\ Department of Polymer Engineering, Budapest University of Technology and Economics, H-1111 Budapest, Hungary
}

Received 18 July 2007; accepted in revised form 23 October 2007

\begin{abstract}
This paper focuses on the effect of the different injection moulding parameters and storing methods on injection moulded thermoplastic maize starch (TPS). The glycerol and water plasticized starch was processed in a twin screw extruder and then with an injection moulding machine to produce TPS dumbbell specimens. Different injection moulding set-ups and storing conditions were used to analyse the effects on the properties of thermoplastic starch. Investigated parameters were injection moulding pressure, holding pressure, and for the storage: storage at 50\% relative humidity, and under ambient conditions. After processing the mechanical and shrinkage properties of the manufactured TPS were determined as a function of the ageing time. While conditioning, the characteristics of the TPS changed from a soft material to a rigid material. Although this main behaviour remained, the different injection moulding parameters changed the characteristics of TPS. Scanning electron microscope observations revealed the changes in the material on ageing.
\end{abstract}

Keywords: biodegradable polymers, thermoplastic starch, injection moulding

\section{Introduction}

In recent years, the research and development on biodegradable polymers has accelerated. This progress is due to the interest of using renewable, natural resources for certain applications in the field of polymer processing [1]. The need for using more renewable resources has grown, because of the shrinking oil reserves, and the environmental friendly consciousness. Biodegradable polymers or biopolymers are capable of biodegradation in certain environments e.g. during composting. These materials represent the highest level of recycling. Biopolymers can be divided into to two main groups [2]. One group contains the biodegradable polyesters, which are petroleum based, but they are biodegradable. The other group contains polymers from renewable resources, like thermoplastic starch or poly-lactic-acid (PLA). Both TPS and PLA can be produced from common, natural resource, and they are readily biodegradable.

Starch can be found in different plants like wheat, maize, potato, rice. This material is in abundance all over the world. Starch has a granular structure, which is built by two main carbohydrate polymers: the linear amylose and the highly branched amylopectin. According to the botanical source the amylose-amylopectin ratio can be different. The granular structure must be totally destructured in order to produce thermoplastic starch. Starch can be processed into thermoplastic starch under the action of both temperature and shearing action [3-5]. Plasticizers like glycerol or water have to be used while processing, because of the low decomposition temperature of the granular starch. Processing usually takes place in an extruder, where the starch granules are disintegrated. After that thermoplastic starch pellets are produced, TPS can

*Corresponding author, e-mail: kovacs@pt.bme.hu

(C) BME-PT and GTE 
be processed into a biodegradable product by injection moulding or compression moulding.

Although starch is a low-cost material, it has some drawbacks, while using it as a material for biodegradable products. One drawback is the so-called retrogradiation or ageing. Many authors reported this behaviour of starch. While ageing, the crystallinity of starch increases, which results in higher stress at elongation and tensile modulus [6-9]. Starch has unfortunately a hydrophilic character, which also retards its widespread industrial applications. While in contact with water, pure starch products swell and then dissolve. Another factor is the low mechanical properties of starch and the high shrinkage values when injection moulded. Although starch possesses these drawbacks, it is a potential material of biodegradable products in the near future. To overcome these drawbacks, a great number of experiments were taken. Many authors reported the behaviour of starch reinforced with natural fibres, like flax, cellulose, jute, ramie, etc. [10-15]. With the reinforcement, the mechanical properties of starch improved, while the ageing, the hydrophilic character, and the shrinkage values decreased. Many authors also reported the behaviour of a starch-based blend. In this case starch was associated with another biopolymer, often with biodegradable polyesters, like polycaprolactone (PCL), polyesteramide (PEA), or with the starch based polylacticacid (PLA) [16-21]. The blending of starch with biodegradable polyesters also decreased the hydrophilic behaviour and the shrinkage values, but it had no effect on the ageing of starch. The mechanical properties of blends vary with the starch-biodegradable polyester content, but unfortunately only some of them are compatible with starch. In recent years, the researches concentrated on the cross linking of starch, and the association of starch with nano-particles [22-27]. With the help of cross-linking or nano-particles, the ageing, and strong hydrophilic character of thermoplastic starch can be reduced.

Starch plays the role of the matrix material in all these biocomposites, constituting the majority component of these blends. When injection moulded, the different injection moulding parameters could affect the long-term behaviour of TPS. This paper focuses on the effect of different injection moulding set-ups on the time dependent behaviour of TPS.

\section{Experimental}

Maize starch was used for the experiments. According to the manufacturer, the amylose-amylopectin ratio is $25: 75$. Maize starch was plasticized with glycerol (99\% purity) and distilled water. The starch:glycerol:water ratio was 70:16:14 and glycerol-mono-stearate was added as a lubricant. A special silicone based screw cleaner material was used to clean the screw of the extruder and injection moulding machine from any contamination (provided by Szilor Ltd., Hungary). The TPS was produced in a two step process. First, the materials were weighted and mixed, than the mixture was processed with a Brabender Plasti-Corder PL 2100 double screw extruder (diameter $25 \mathrm{~mm}$ screw, $L / D=20$ ) with zone temperatures of 90-130-120$90^{\circ} \mathrm{C}$ (from hopper to die). The extrudate was granulated, and stored under controlled conditions (50\% relative humidity for 4 weeks) before injection moulded. After the storing process, the granuletes were injection moulded with an Arburg 320 600$250 \mathrm{C}$ injection moulding machine equipped with a diameter $35 \mathrm{~mm}$ screw. Table 1 contains the main parameters of the injection moulding process.

Dumbbell test specimens were injection moulded for the tensile and shrinkage measurements. The injection moulding pressure was 1400 bars, and the holding pressure was varied from 600 to 1000 bars to determine the effect of the holding pressure on the time dependence of TPS (Table 2). The injection moulded specimens were stored different ways (at 50\% relative humidity and under ambient conditions) and for different time periods to determine the effect of ageing time and different storage type (Table 2).

Table 1. The main injection moulding parameters used

\begin{tabular}{|l|c|c|}
\hline \multicolumn{3}{|c|}{ Injection moulding parameters } \\
\hline Injection volume & 44 & {$\left[\mathrm{~cm}^{3}\right]$} \\
\hline Injection pressure & 1400 & {$[\mathrm{bar}]$} \\
\hline Switch over point & 12 & {$\left[\mathrm{~cm}^{3}\right]$} \\
\hline Injection rate & 50 & {$\left[\mathrm{~cm}^{3} / \mathrm{s}\right]$} \\
\hline Holding pressure & VARIED $(600-1000)$ & {$[\mathrm{bar}]$} \\
\hline Holding pressure & 20 & {$[\mathrm{~s}]$} \\
\hline Cooling time & 115 & {$[\mathrm{~s}]$} \\
\hline & 115 & {$\left[{ }^{\circ} \mathrm{C}\right]$} \\
\hline 1. Zone & 120 & {$\left[{ }^{\circ} \mathrm{C}\right]$} \\
\hline 2. Zone & 125 & {$\left[{ }^{\circ} \mathrm{C}\right]$} \\
\hline 3. Zone & 130 & {$\left[{ }^{\circ} \mathrm{C}\right]$} \\
\hline 4. Zone & 25 & {$\left[{ }^{\circ} \mathrm{C}\right]$} \\
\hline 5. Zone & {$\left[{ }^{\circ} \mathrm{C}\right]$} \\
\hline Mould temperature &
\end{tabular}


Table 2. The investigated parameters

\begin{tabular}{|c|c|c|}
\hline & $\begin{array}{c}\text { 800 bars of } \\
\text { holding pressure }\end{array}$ & $\begin{array}{c}\text { Varied } \\
\text { holding pressure }\end{array}$ \\
\hline $\begin{array}{c}50 \% \text { relative } \\
\text { humidity }\end{array}$ & $\times$ \\
\hline $\begin{array}{c}\text { Varied } \\
\text { conditions }\end{array}$ & $\times$ & \\
\hline
\end{tabular}

Tensile tests (according to standard EN ISO 5275:1999) and shrinkage measurement (according to standard EN ISO 2944:2003) were taken at 1-24-24-48-72-168 hours after the injection moulding. A crosshead speed of $50 \mathrm{~mm} / \mathrm{min}$ was used to determine the tensile properties. Finally, scanning electron microscope (SEM) observations were taken to determine the changes in the TPS material as a function of ageing time.

\section{Results and discussion}

Figure 1 and 2 show the evolution of the tensile stress and tensile modulus as the function of the ageing time (holding pressure was 800 bars).

Two different storing methods can be distinguished: storing at 50\% relative humidity and storing under ambient conditions. Ambient conditions mean that the specimens were stored indoor, in free air (at $40 \% \pm 10 \%$ relative humidity, UV degradation or contact with rain excluded). As it can be observed the type of storage has great effect on the properties of TPS. Storing under ambient conditions accelerates the ageing of TPS, while storing under controlled conditions retarded the ageing. Although the tensile properties increased in time, the strain decreased (Figure 3).

The strain decrease of the specimens stored under ambient conditions is much more intense than the

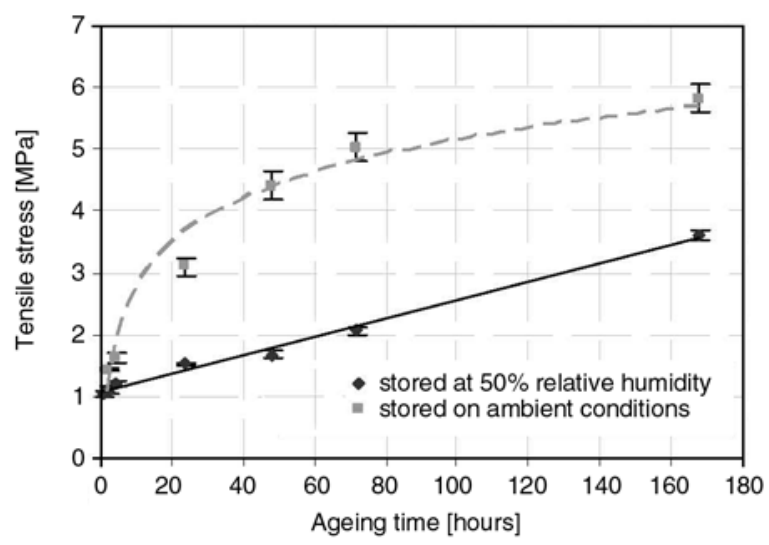

Figure 1. The effect of the different storing conditions on the tensile stress

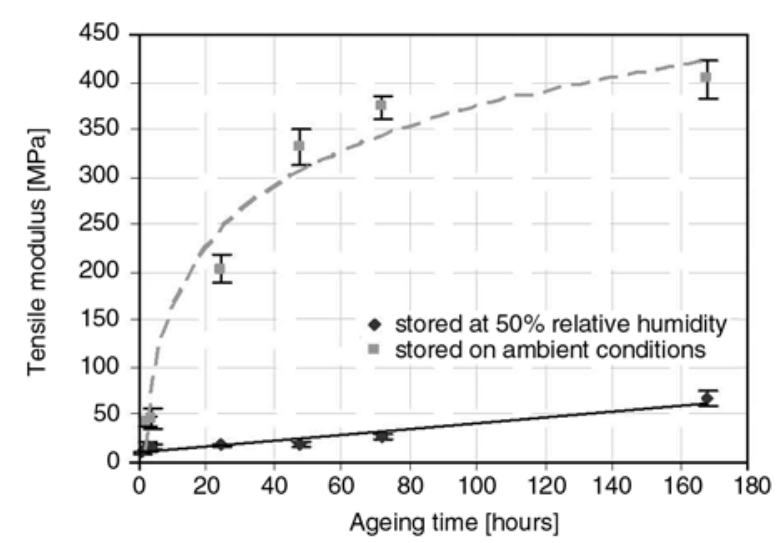

Figure 2. The effect of the different storing conditions on the tensile modulus

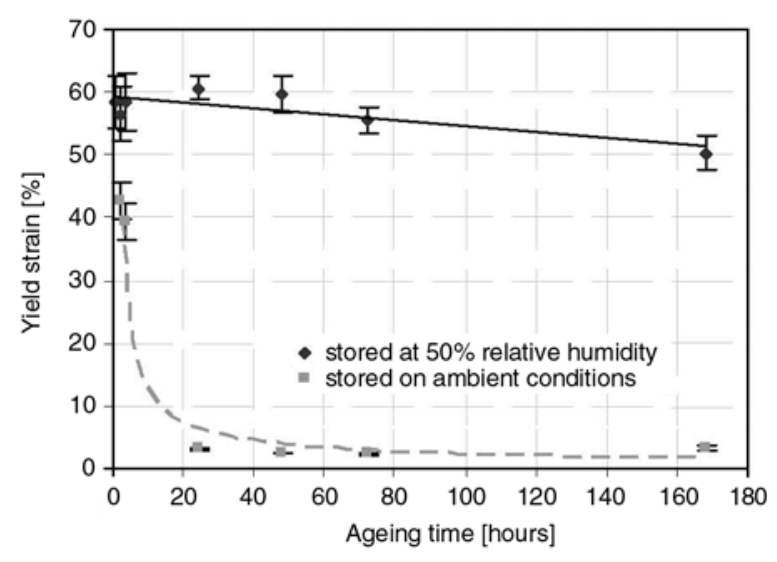

Figure 3. The effect of the different storing conditions on the yield strain

strain decrease of the specimens stored at 50\% relative humidity.

In time the properties of the material changed from soft to rigid behaviour, as it can be observed on Figure 4.

The holding pressure was varied from 600 to 1000 bars to determine whether this parameter has

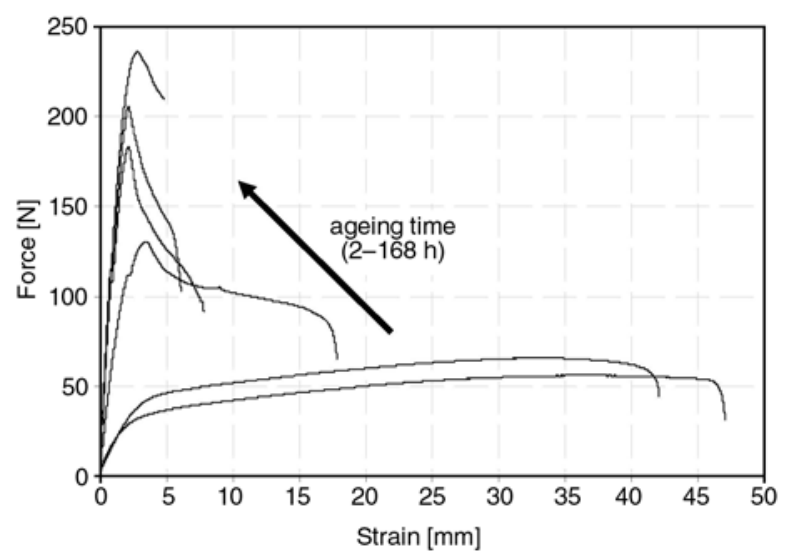

Figure 4. The change of tensile diagrams as a function of ageing time (2-4-24-48-72-168 hours, at ambient conditions) 


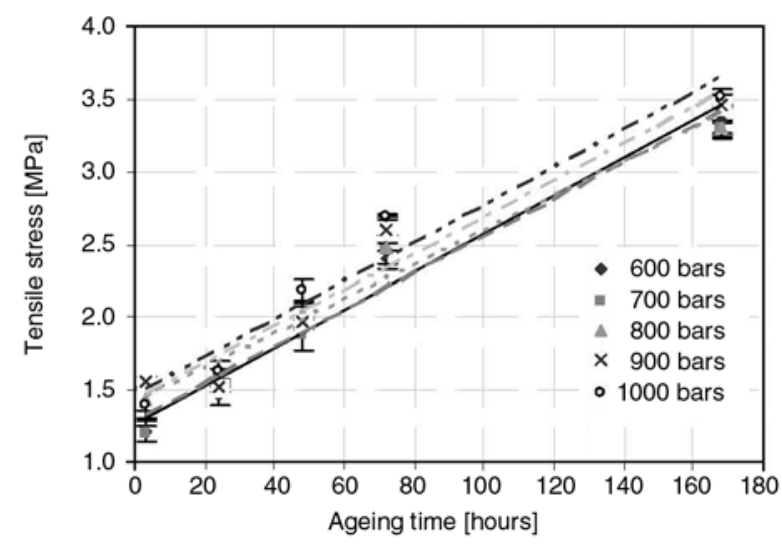

Figure 5. The effect of ageing time on the tensile stress

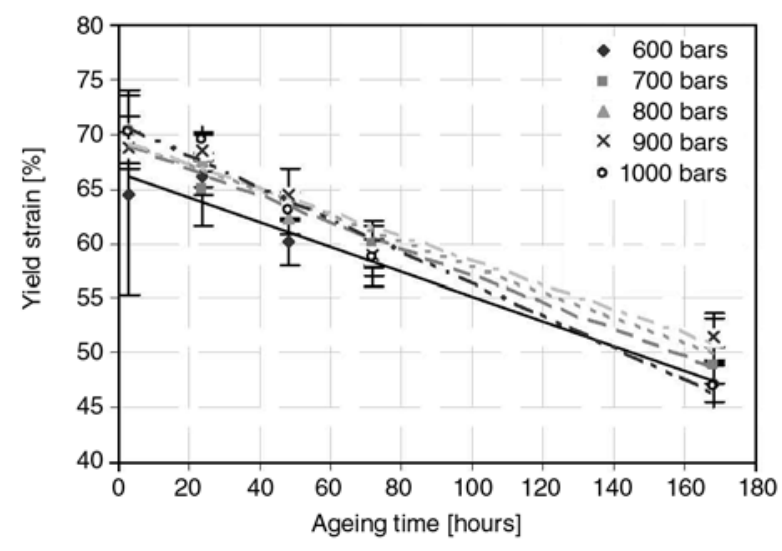

Figure 6. The effect of ageing time on the yield strain

any effect on the time dependent behaviour of TPS or not. The holding pressure had almost no effect on the tensile stress and tensile strain, as it can be seen in Figure 5 and 6.

While the tensile stress increased, the yield strain decreased independently from the holding pressure. The holding pressure had major effect on the tensile modulus (Figure 7) in time.

Although the tensile modulus of the TPS was the same just after injection moulding on all the hold-

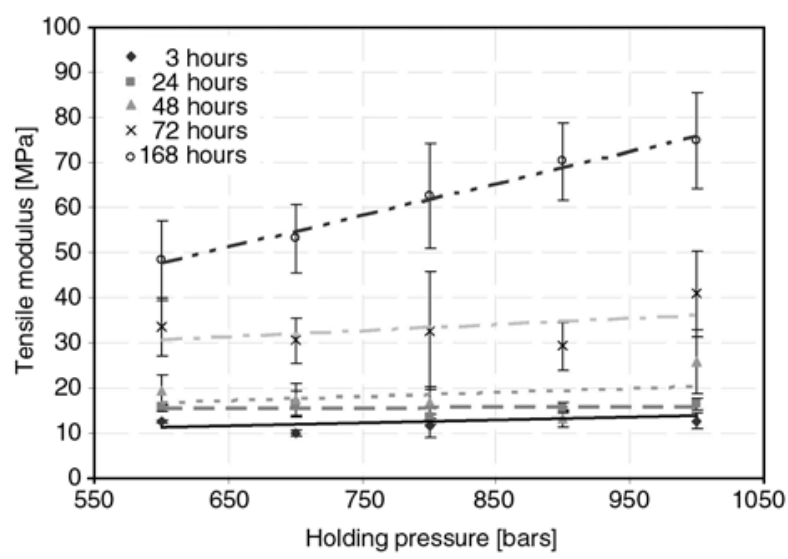

Figure 7. The effect of ageing time on the tensile modulus

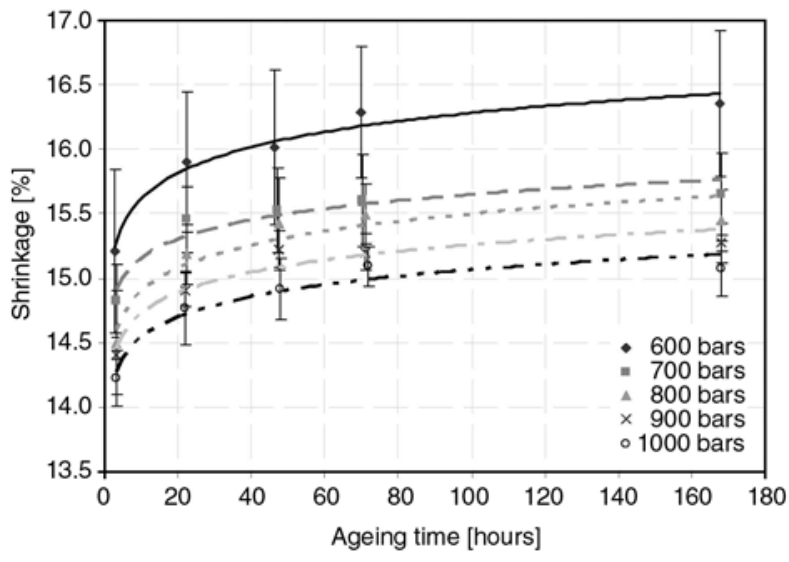

Figure 8. Linear shrinkage as a function of the ageing time

ing pressures, the holding pressure had effect on the time dependent behaviour of TPS. The calculated correlation coefficients demonstrate that even 72 hours after the ejection, the holding pressure history of the specimens had almost no effect on the tensile modulus. 168 hours after the demoulding, the holding pressure history of the specimens was not ignorable, the more the holding pressure applied, the more the measured tensile modulus values raised (even though the scatter was relatively high). This phenomenon is to be further researched in following papers. The effect of the holding pressure on the shrinkage properties can be monitored on Figure 8. Although the shrinkage of TPS is high, it can be lowered by applying higher holding pressure values.

The SEM images revealed that after the first extrusion the TPS pellets still contain some granular starch (Figure 9), which is almost totally disappeared after the injection moulding (Figure 10) or

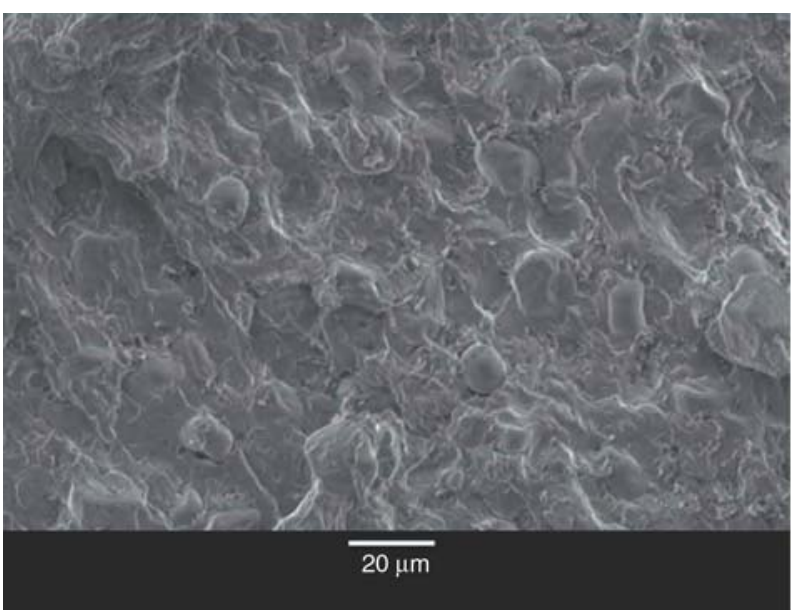

Figure 9. TPS surface after the first extrusion. Native starch pellets still can be found 


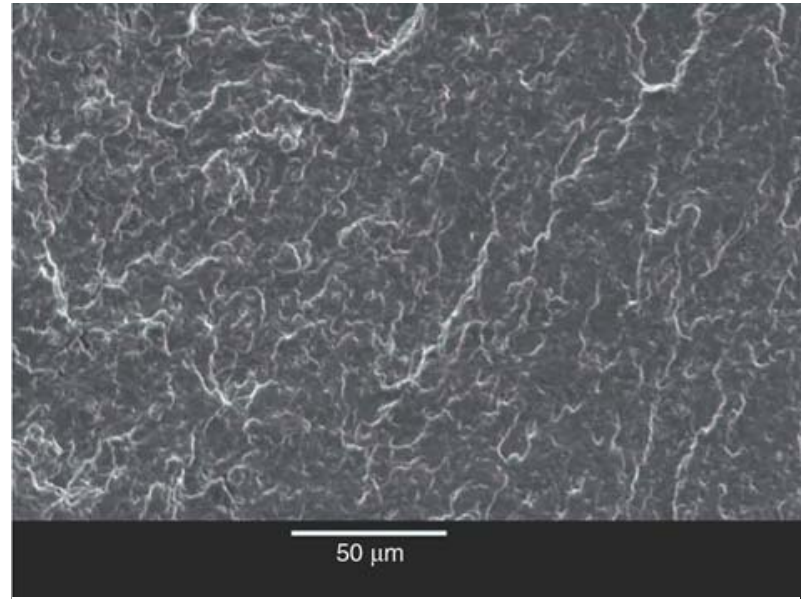

Figure 10. TPS surface after injection moulding. Native starch granules totally destructur

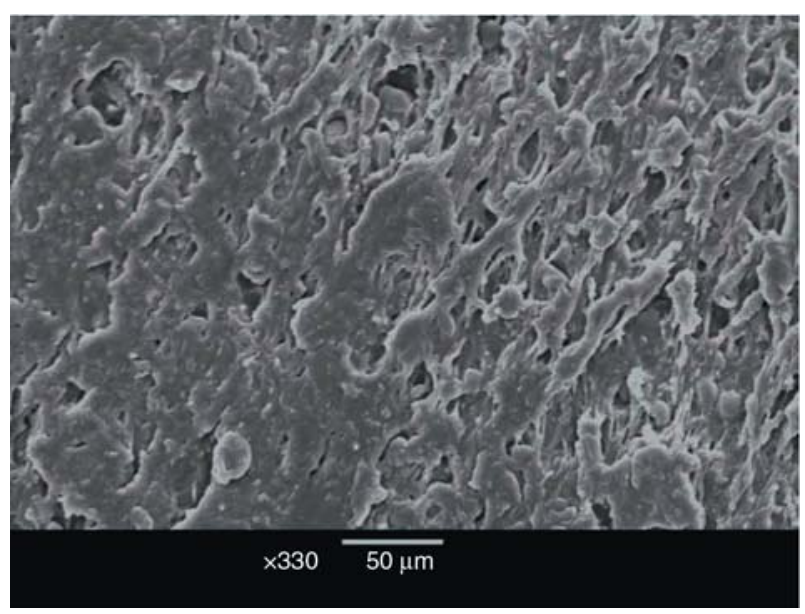

Figure 11. TPS surface after a second extrusion process. Native starch granules totally destructured

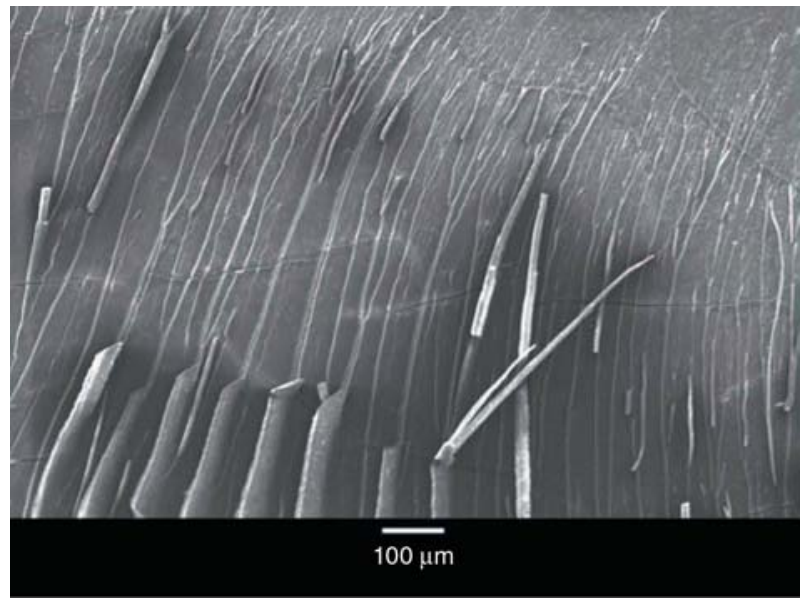

Figure 12. Aged TPS fracture surface. Significant quantity of cracks can be found

the second extrusion process (Figure 11). Although wide angle $\mathrm{X}$-ray scattering measurements were taken (was not published in this paper) which indicated that the starch crystallinity disappeared (gran- ular structure disintegrated), it is inconsistent with the SEM observations.

The SEM images verified that while ageing the material properties changed from rubber like behaviour to rigid. This phenomenon can be observed on the surface of the broken tensile test specimens. The fracture surface of the specimens became smoother and significant quantity of cracks can be detected as a function of ageing (Figure 12).

\section{Conclusions}

Thermoplastic starch (maize starch:glycerol:water ratio is 70:16:14) was produced by extrusion and injection moulding. The effect of various injection moulding parameters and storing methods (on ambient conditions; at 50\% relative humidity) were studied. It was found that ageing occurs much faster when TPS is stored on ambient conditions rather than at $50 \%$ relative humidity. The holding pressure had no effect on the tensile stress and on the yield strain of the specimens, but it had on the tensile modulus. The holding pressure history of the specimens had no effect on the tensile modulus for 72 hours. After this time period the holding pressure history of the specimens was not ignorable: the more the holding pressure applied, the more the tensile modulus raised. This phenomenon will be studied further in the future. The further investigation of holding pressure showed that it lowers the relatively high shrinkage values of TPS. The tensile diagrams of TPS showed that the properties of TPS changed from rubber like to a rigid behaviour. SEM images verified this phenomenon. The surface of the broken bars changed, and a rigid fracture surface was observed after 8 weeks of ageing.

\section{Acknowledgements}

The authors would like to thank Arburg Ltd. for the injection moulding machine, Wittmann Ltd. for mould temperature control unit, Anton Ltd. (Hungary) for the moulds and Szilor Ltd. (Hungary) for the screw cleaner material.

\section{References}

[1] Zhang M. Q.: Polymeric materials from natural resources-emerging as the times require. Express Polymer Letters, 1, 406 (2007). 
[2] Avérous L.: Biodegradable multiphase systems based on plasticized starch: A review. Journal of Macromolecular Science, Part C: Polymer Reviews, 44, 231-274 (2004).

[3] Stepto R. F. T.: Thermoplastic starch. Macromolecular Symposia, 152, 73-82 (2000).

[4] Stepto R. F. T.: The processing of starch as a thermoplastic. Macromolecular Symposia, 201, 203-212 (2003).

[5] Czigány T., Romhány G., Kovács J. G.: Starch for injection moulding purposes. in 'Engineering Biopolymers: Homopolymers, Blends, and Composites' (eds.: Fakirov S., Bhattacharyya D.) Hanser, Munich, 81108 (2007).

[6] Van Soest J. J. G., Hulleman S. H. D., De Wit D., Vliegenthart J. F. G.: Crystallinity in starch bioplastics. Industrial Crops and Products, 5, 11-22 (1996).

[7] Van Soest J. J. G., Vliegenthart J. F. G.: Crystallinity in starch plastics: Consequences for material properties. Trends in Biotechnology, 15, 208-213 (1997).

[8] Van Soest J. J. G., Hulleman S. H. D., De Wit D., Vliegenthart J. F. G.: Changes in the mechanical properties of thermoplastic potato starch in relation with changes in B-type crystallinity. Carbohydrate Polymers, 29, 225-232 (1996).

[9] Kuutti L., Peltonen J., Myllärinen P., Teleman O., Forssell P.: AFM in studies of thermoplastic starches during ageing. Carbohydrate Polymers, 37, 7-12 (1998).

[10] Wollerdorfer M., Bader H.: Influence of natural fibres ont he mechanical properties of biodegradable polymers. Industrial Crops and Products, 8, 105-112 (1998).

[11] Averous L., Boquillon N.: Biocomposites based on plasticized starch: thermal and mechanical behaviours. Carbohydrate Polymers, 56, 111-122 (2004).

[12] Avérous L., Fringant C., Moro L.: Plasticized starchcellulose interactions in polysaccharide composites. Polymer, 42, 6565-6572 (2001).

[13] Gáspár M., Benkô Zs., Dogossy G., Réczey K., Czigány T.: Reducing water absorption in compostable starch-based plastics. Polymer Degradation and Stability, 90, 563-569 (2005).

[14] Ma X., Yu J., Kennedy J. F.: Studies on the properties of natural fiber-reinforced thermoplastic starch composites. Carbohydate Polymers, 62, 19-24 (2005).
[15] Bodros E., Pillin I., Montrelay N., Baley C.: Could biopolymers reinforced by randomly scattered flax fibre be used in structural applications? Composites Science and Technology, 67, 462-470 (2007).

[16] Martin O., Avérous L.: Poly(lactic acid): plasticization and properties of biodegradable multiphase systems. Polymer, 42, 6209-6219 (2001).

[17] Avérous L., Moro L., Dole P., Fringant C.: Properties of thermoplastic blends: starch-polycaprolactone. Polymer, 41, 4157-4167 (2000).

[18] Avérous L., Fringant C.: Association between plasticized starch and polyesters: processing and performances of injected biodegradable systems. Polymer Engineering and Science, 41, 727-734 (2001).

[19] Huneault M. A., Li H.: Morphology and properties of compatibilized polylactide/thermoplastic starch blends. Polymer, 48, 270-280 (2007).

[20] Avérous L., Fauconnier N., Moro L., Fringant C.: Blends of thermoplastic starch and polyesteramide: processing and properties. Journal of Applied Polymer Science, 76, 1117-1128 (2000).

[21] Schwach E, Avérous L.: Starch-based biodegradable blends: Morphology and interface properties. Polymer International, 53, 2115-2124 (2004).

[22] Huang M-f., Yu J-g., Ma X-f., Jin P.: High performance biodegradable thermoplastic starch-EMMT nanoplastics. Polymer, 46, 3157-3162 (2005).

[23] Huang M., Yu J., Ma X.: High mechanical performance MMT-urea and formamide-plasticized thermoplastic cornstarch biodegradable nanocomposites. Carbohydrate Polymers, 63, 393-399 (2006).

[24] Huang M-F., Yu J-G., Ma X-F.: Studies on the properties of montmorillonite reinforced thermoplastic starch composites. Polymer, 45, 7017-7023 (2004).

[25] Delville J., Joly C., Dole P., Bliard C.: Influence of photocrosslinking on the retrogradiation of wheat starch based films. Carbohydrate Polymers, 53, 373381 (2003).

[26] Zhai M., Yoshii F., Kume T.: Radiation modification of starch-based plastic sheets. Carbohydrate Polymers, 52, 311-317 (2003).

[27] Silva M. C., Ibezim E. C., Ribeiro T. A. A., Carvalho C. W. P., Andrade C. T.: Reactive processing and mechanical properties of cross-linked maize starch. Industrial Crops and Products, 24, 46-51 (2006). 